\title{
Rational Engineering of Diol Dehydratase Enables 1,4-Butanediol Biosynthesis From
}

\section{Xylose}

Jia Wang a, b, $¥$, Rachit Jain ${ }^{c, ¥}$, Xiaolin Shen ${ }^{a, b}$, Xinxiao Sun ${ }^{a, b}$, Mengyin Cheng ${ }^{c}$, James C.

$$
\text { Liao }^{\text {d, e }} \text {, Qipeng Yuan a, b, *, Yajun Yan }{ }^{c} \text { * }
$$




\begin{abstract}
Establishing novel synthetic routes for microbial production of chemicals often requires overcoming pathway bottlenecks by tailoring enzymes to enhance bio-catalysis or even achieve non-native catalysis. Diol dehydratases have been extensively studied for their interactions with C2 and C3 diols. However, attempts on utilizing these insights to enable catalysis on non-native substrates with more than two hydroxyl groups have been plagued with low efficiencies. Here, we rationally engineered the Klebsiella oxytoca diol dehydratase to enable and enhance catalytic activity toward a non-native C4 triol, 1,2,4-butanetriol. We analyzed dehydratase's interaction with 1,2-propanediol and glycerol, which led us to develop rationally conceived hypotheses. An in silico approach was then developed to identify and screen candidate mutants with desired activity. This led to an engineered diol dehydratase with nearly 5 fold higher catalytic activity toward 1,2,4-butanetriol than the wild type as determined by in vitro assays. Based on this result, we then expanded the 1,2,4-butanetriol pathway to establish a novel 1,4-butanediol production platform. We engineered Escherichia coli's xylose catabolism to enhance the biosynthesis of 1,2,4-butanetriol from $224 \mathrm{mg} / \mathrm{L}$ to $1506 \mathrm{mg} / \mathrm{L}$. By introducing the complete pathway in the engineered strain we achieve de novo biosynthesis of 1,4-butanediol at $209 \mathrm{mg} / \mathrm{L}$ from xylose. This work expands the repertoire of substrates catalyzed by diol dehydratases and serves as an elucidation to establish novel biosynthetic pathways involving dehydratase based biocatalysis.
\end{abstract}

Keywords: 1,4-Butanediol, Diol dehydratase, Xylose Metabolism, 1,2,4-Butanetriol, Protein Engineering 


\section{Introduction}

Biological platform for production of various industrially attractive chemicals including pharmaceuticals (Ajikumar et al., 2010; Lin et al., 2014; Ro et al., 2006; Yan et al., 2008), biofuels (Atsumi et al., 2008; Avalos et al., 2013; Huo et al., 2011; Lan et al., 2013; Xu et al., 2013), polymers (Jung and Lee, 2011; Nikel et al., 2010; Xiong et al., 2014) and bulk chemicals (Jang et al., 2012; Raynaud et al., 2003; Shen et al., 2012) has been established by introducing synthetic routes or enhancing native routes (Ohta et al., 1991; Sun et al., 2015; Vemuri et al., 2002) in microbes.

1,4-Butanediol (1,4-BDO) is a valuable commodity chemical (annual market of over 2.5 million tons worldwide), which is not known to be natively produced by any microbe. It is used in the manufacture of plastics, solvents, fibers, other chemicals whose combined market is in excess of \$4 billion (Ji and Huang, 2014; Yim et al., 2011). Hence, the production of 1,4-BDO from biological processes holds prominent commercial value. Direct production of 1,4-BDO via metabolically engineered E. coli was firstly achieved in 2011 (Yim et al., 2011). In this pathway, two E. coli native enzymes and four exogenous enzymes were over-expressed to convert the TCA cycle key intermediate succinate into 1,4-BDO. This pathway has a maximum theoretical yield of $0.545 \mathrm{~g}$ 1,4-BDO/g glucose as reported (Yim et al., 2011). Systematic optimization of the whole pathway enabled $18 \mathrm{~g} / \mathrm{L}$ of 1,4-BDO produced from glucose. In order to improve the efficiency of this pathway, the mutated butyraldehyde dehydrogenase (encoded by Bld) and butanol dehydrogenase (encoded by bdh) from Clostridium saccharoperbutylacetonicum were used to replace the rate-limiting enzyme alcohol dehydrogenase (encoded by adhE2) (Hwang et 
al., 2014). The best strain produced 1,4-BDO titers 4 folds higher than the wild type bld. Despite the higher titers achieved, this pathway contains multiple reaction steps (totally 21 steps) and suffers complicated regulations. Recently, an alternative 1,4-BDO biosynthesis pathway has been reported (Liu and Lu, 2015; Tai et al., 2016). This pathway bypasses the TCA cycle and assimilates the lignocellulose-derived sugars into 1,4-BDO in less than six steps. As reported, this pathway has a maximum theoretical yield of $0.6 \mathrm{~g} 1,4-\mathrm{BDO} / \mathrm{g}$ xylose, when additional reducing power can be provided by other energy sources. The titer of xylose-derived 1,4-BDO reached $3.83 \mathrm{~g} / \mathrm{L}$ in shake flasks with a $63 \%$ of theoretical yield. However, the major by-product 1,2,4-butanetriol also accumulated up to $1 \mathrm{~g} / \mathrm{L}$ (Tai et al., 2016).

In this work, we established a novel microbial route for the production of 1,4-BDO by introducing a synthetic pathway in Escherichia coli and engineering its xylose catabolism (Fig. 1), which has the same maximum theoretical yield as the above 1,4-BDO pathway from xylose. In our proposed process, the dehydration of 1,2,4-butanetriol $(1,2,4-\mathrm{BTO})$ represents the bottleneck for 1,4-BDO production. In order to overcome this bottleneck, we developed rational protein engineering strategies to enhance the activity of Klebsiella oxytoca diol dehydratase toward the non-native substrate 1,2,4-BTO.

The K. oxytoca diol dehydratase has a complex coenzyme B12 dependent reaction mechanism, and is known to dehydrate its native substrate - 1,2-propanediol (1,2-PD) efficiently and to undergo suicide inactivation in the presence of a C3 triol - glycerol (Doitomi et al., 2012). Since the catalytic mechanism of $K$. oxytoca diol dehydratase has been well studied with 1,2-PD and 
also with glycerol, we chose this enzyme as a candidate for rational engineering approaches to promote catalysis toward a non-native $\mathrm{C} 4$ triol - 1,2,4-BTO.

Rational approaches to engineer protein have been largely pursued via manipulation of amino acid residues in the catalytic pocket achieved via site directed mutagenesis (Marcheschi et al., 2013). In order to promote non-native catalysis toward larger substrates, increasing the catalytic pocket has been the predominant strategy, generally achieved via replacement of target residues with smaller ones (Marcheschi et al., 2013). Additionally, substituting target residues via saturation mutagenesis (Savile et al., 2010) and/or directed evolution (Kan et al., 2016) have been shown to improve catalysis possibly due to changes in the above mentioned interactions altering catalysis. The utilization of these semi-rational approaches are in turn contingent on sensitivity of high-throughput screening platforms for identification of optimal mutant(s) which generally involves laborious experimental procedures (Dietrich et al., 2010).

Here, we engineered the $K$. oxytoca diol dehydratase to achieve non-native catalysis via a twostep rational design approach taking into account critical interactions influencing catalysis. We first adapted predominant strategies of rational design via preventing interaction with an undesired residue and increasing the size of the catalytic pocket to enable the accommodation of a larger substrate. In the second step of rational design, we studied the effect of mutating target amino acids in the catalytic pocket on substrate position based upon steric effects while maintaining a relatively similar local hydrophobic environment. Prudent application of this approach led to increasing dehydratase activity by nearly 5 folds toward 1,2,4-BTO in 
comparison to the native protein. These efforts enabled the production of 1,4-butanediol $(1,4-$ BDO) via a novel metabolic route (Fig. 1) from xylose, which is different from the previous artificial pathway commencing from succinate and/ or $\alpha$-ketoglutarate (Hwang et al., 2014; Yim et al., 2011). 


\section{Materials and Methods}

\subsection{Chemicals and reagents}

1,2,4-BTO, 1,4-BDO, 1,3-propanediol standards were procured from Alfa Aesar (Ward Hill, MA); 1,2-PD was procured from Sigma Aldrich (St. Louis, MO); 1-propanol was procured from Fisher Scientific (Atlanta, GA). All enzymes and kits used for DNA manipulation (Restriction enzymes, Phusion high fidelity DNA polymerase and Quick DNA ligase) were procured from New England Biolabs (Beverly, MA).

\subsection{Bacterial strains and plasmids}

Whole cell bio-catalysis studies and de novo biosynthesis studies were carried out using E. coli BW25113 (E. coli Genetic Resource Center). Knock-out strains of E. coli BW25113 were constructed by disrupting target gene(s) using P1 transduction as described previously (Thomason et al., 2007). Loss of antibiotic resistance was facilitated by transferring pCP20 into target strains (Datsenko and Wanner, 2000). Colony PCR was used to verify the removal of antibiotic resistance using GoTaq DNA polymerase (Promega, WI). E. coli BL21 Star (DE3) (Invitrogen, CA) was used for protein expression in enzyme assay experiments. E. coli XL1-Blue (Stratagene, CA) served as the cloning host for DNA manipulation experiments. The cloning plasmids used were pZE12-luc (Lutz and Bujard, 1997) and pCS27 (Shen and Liao, 2008) for whole cell bio-catalysis studies and shake flask experiments; pETDuet-1 (EMD Chemicals Inc., NJ) for his-tag cloning and protein purification experiments. 


\subsection{DNA manipulations}

Plasmids constructed in this work are listed in Table 1. The $K$. oxytoca diol dehydratase reactivase factors $(d d r A B)$ were cloned into the backbone of pCS27 using Acc65I, BsiWI and HindIII to create pRJ75. The $K$. oxytoca fusion diol dehydratase ( $p p d A-C-B$ ) reading frame was cloned into the backbone of pZE12-luc using EcoRI and XbaI to create pJW79. The general procedure to clone diol dehydratase mutants into pZE12-luc (pJW80-83, pRJ111-116, and pRJ161-167) is described below. In order to create single amino acid mutations, overlap PCR was performed using appropriate primers and pJW79 as the template. The resultant PCR products were purified and cloned into the backbone of pZE12-luc using EcoRI and XbaI. This procedure was repeated in order to create more than one amino acid mutations. All mutants were sequenced (Eurofins) to verify the accuracy of mutations.

In order to purify diol dehydratase protein and its mutants, the fusion $p p d A-C-B$ reading frame and its mutants were cloned into his-tag plasmid (pETDuet-1) using BamHI and XhoI (pRJ84, pRJ91, pRJ170). Similarly, E. coli $y q h D$ was cloned into the backbone of pETDuet-1 using BamHI and PstI to create pRJ122. The genes $x y l B$ and $x y l C$ from Caulobacter crescentus were codon-optimized for expression in E. coli and synthesized by GENEWIZ (South Plainfield, NJ). The codon optimized $x y l B, x y l C$ genes and $y q h D$ gene were cloned into the backbone of pCS27 using Acc65I, PstI and SalI to create pJW60. The gene $x y l D$ from C. crescentus and kivD gene from Lactococcus lactis were cloned into the backbone of pZE12-luc simultaneously using Acc65I, PstI and XbaI to create pJW76. The genes $x y l D, k i v D$ and fusion $p p d A-C-B$ were cloned simultaneously into pZE12-luc using Acc65I, PstI, BamHI and XbaI to create pJW84. Plasmids 
pJW85 and pJW86 were constructed similarly as pJW84, however with the S301AQ336A or S301AQ336AV300M mutants of $p p d A-C-B$ respectively.

\subsection{Culture medium}

Modified M9 medium was used for whole bio-catalysis, while LB xylose medium was used for de novo biosynthesis experiments. The modified M9 medium consisted of (per liter): $20 \mathrm{~g}$ glucose, $12.8 \mathrm{~g} \mathrm{Na}_{2} \mathrm{HPO}_{4} .7 \mathrm{H}_{2} \mathrm{O}, 3 \mathrm{~g} \mathrm{KH}_{2} \mathrm{PO}_{4}, 1 \mathrm{~g} \mathrm{NH}{ }_{4} \mathrm{Cl}, 0.5 \mathrm{~g} \mathrm{NaCl}, 0.5 \mathrm{mM} \mathrm{MgSO} 4,0.05 \mathrm{mM}$ $\mathrm{CaCl}_{2}$ and $5 \mathrm{~g}$ yeast extract (Jain et al., 2014). LB xylose medium consisted of LB media supplemented with $20 \mathrm{~g} / \mathrm{L}$ xylose.

\subsection{Whole cell bio-catalysis}

Whole cell bio-catalysis experiments were performed with modifications to the method described previously (Jain and Yan, 2011). Native or mutant dehydratases were cloned into pZE12-luc and the wild type E. coli BW25113 was transformed with each of those plasmids. Overnight cultures $(3 \mathrm{~mL})$ of transformants were grown in $\mathrm{LB}\left(37^{\circ} \mathrm{C}, 250 \mathrm{rpm}\right)$ and served as the pre-inoculum. The experiments were carried out in $125 \mathrm{~mL}$ screw cap bottles with $10 \mathrm{~mL}$ of the modified M9 medium. About $100 \mu \mathrm{L}$ of the pre-inoculum was added to the screw cap bottles along with appropriate antibiotics and grown for 4 hours $\left(37^{\circ} \mathrm{C}, 250 \mathrm{rpm}\right)$. Following this, $10 \mu \mathrm{M}$ coenzyme B12, $0.1 \mathrm{mM}$ IPTG and $1 \mathrm{~g} / \mathrm{L}$ substrate (1,2-PD/ 1,2,4-BTO/ glycerol) were added to the cultures. The cultures were then grown for another 24 hours $\left(37^{\circ} \mathrm{C}, 250 \mathrm{rpm}\right)$. HPLC analysis was done at the end of the study for product analysis by collecting $1 \mathrm{~mL}$ samples.

\subsection{In vitro coupled enzyme assays}


In order to determine the enzyme kinetics $\left(K_{m}, K_{\text {cat }}\right)$ of diol dehydratase, fusion $p p d A-C-B$ and its mutants were first cloned into pETDuet-1 plasmid, respectively. Additionally, the E. coli gene encoding alcohol dehydrogenase $y q h D$ was also cloned into pETDuet-1 plasmid, separately. The constructed plasmids were then transferred separately into E. coli BL21 Star (DE3). Overnight culture of transformants were made in $3 \mathrm{~mL} \mathrm{LB}$, grown overnight at $37^{\circ} \mathrm{C}, 250 \mathrm{rpm}$. Following this, $500 \mu \mathrm{L}$ of seed cultures were added to $125 \mathrm{~mL}$ shake flasks containing $50 \mathrm{~mL}$ LB. The cultures were then grown at $37^{\circ} \mathrm{C}$ until the optical density at $600 \mathrm{~nm}$ reached 0.6 , following which $1 \mathrm{mM} \mathrm{IPTG}$ was added and cells were grown for another 5 hours at $30^{\circ} \mathrm{C}$. In order to obtain the purified protein, the cultures were first centrifuged to concentrate the cells. Using a Mini Bead Beater (Biospec), the cells were broken and the mixture was centrifuged again. The resultant supernatant was purified using His-Spin Protein Miniprep kit (Zymo Research, Irvine, $\mathrm{CA}$ ) and the protein concentration was determined using Pierce BCA protein assay kit (Thermo Scientific, Atlanta, GA).

Diol dehydratase activity was monitored via coupling the dehydration activity with the subsequent alcohol dehydrogenase activity. The reaction mixture consisted of $0.2 \mathrm{mM}$ coenzyme B12, $0.035 \mathrm{M} \mathrm{K}_{2} \mathrm{HPO}_{4}(\mathrm{pH} 8.0), 0.025 \mathrm{M} \mathrm{KCl}, 0.5 \mathrm{mM} \mathrm{NADPH}, 5 \mathrm{mM} \mathrm{ZnCl}$, purified diol dehydratase protein $(0.02 \mathrm{mg} / \mathrm{mL})$, purified alcohol dehydrogenase protein $(y q h D)$ in excess $(0.11 \mathrm{mg} / \mathrm{mL})$. The reaction was begun with the addition of $0-20 \mathrm{mM}$ substrate $(1,2-\mathrm{PD} / 1,2,4-$ BTO). The total reaction volume was maintained at $1 \mathrm{~mL}$ and the rate of consumption of NADPH was monitored at $340 \mathrm{~nm}$ for $1 \mathrm{~min}$ at room temperature. Appropriate controls were used (without substrate/ without purified protein(s)/ without NADPH). Assay results were 
analyzed $\left(\mathrm{n}=3\right.$ ) via non-linear curve regression using origin lab software to determine $K_{m}$ values. Each data point for non-linear curve regression was the average value of three independent experiments. The standard errors were provided by origin lab software when conducting nonlinear curve regression. To calculate $K_{\text {cat }}$, the molecular weight of protein used was $104720 \mathrm{Da}$.

\subsection{De novo biosynthesis}

Shake flask experiments were performed using LB xylose medium with modifications to the method described previously (Jain and Yan, 2011). Overnight cultures (3 mL) of transformants were grown in $\mathrm{LB}\left(37^{\circ} \mathrm{C}, 250 \mathrm{rpm}\right)$ and served as the pre-inoculum. Screw cap bottles $(125 \mathrm{~mL})$ consisting of $20 \mathrm{~mL}$ LB xylose medium were used for de novo biosynthesis experiments. About $200 \mu \mathrm{L}$ of the pre-inoculum was added to the screw cap bottles along with appropriate antibiotics and grown for 4 hours $\left(37^{\circ} \mathrm{C}, 250 \mathrm{rpm}\right)$. Following this, $10 \mu \mathrm{M}$ coenzyme B12 and $0.1 \mathrm{mM}$ IPTG were added to the cultures. The cultures were then grown for another 48 hours (unless mentioned otherwise) at $37^{\circ} \mathrm{C}, 250 \mathrm{rpm}$. HPLC analysis was done at the end of the study by collecting $1 \mathrm{~mL}$ samples.

\subsection{Analytical procedures}

Optical density was measured at $600 \mathrm{~nm}$ using a Genesys 10S UV-Vis Spectrophotometer (Thermo Scientific, Waltham, MA). For product quantification and separation, $1 \mathrm{~mL}$ samples were collected at the end of whole cell bio-catalysis and de novo biosynthesis experiments. The samples were centrifuged at $15,000 \mathrm{rpm}$ for $12 \mathrm{~min}$, following which the supernatant was filtered and analyzed (Jain et al., 2014). For quantification of organic acids (Eiteman and Chastain, 
1997), 1,4-BDO, 1,2,4-BTO, 1,2-PD, 1-propanol and 1,3-propanediol, HPLC-RID (Shimadzu) with Coregel-64H column (Transgenomic) was used. The eluent used was $4 \mathrm{mN} \mathrm{H}_{2} \mathrm{SO}_{4}$ and the column temperature was set at $60^{\circ} \mathrm{C}$ for separation of xylose, 1,2-PD, 1-propanol, 1,3propanediol and glycerol. For the separation of 1,2,4-BTO and 1,4-BDO the column temperature was set at $40^{\circ} \mathrm{C}$ and the eluent used was $16 \mathrm{mN} \mathrm{H}_{2} \mathrm{SO}_{4}$.

\subsection{Computational analysis and simulations}

The crystal structures used for analysis of $K$. oxytoca diol dehydratase were obtained from Protein Data Bank and included the following: substrate free form (PDBid: 1IWB) (Shibata et al., 2002), bound to 1,2-PD (PDBid: 1UC5) (Shibata et al., 2003) and bound to glycerol (PDBid: 3AUJ) (Yamanishi et al., 2012). Docking studies and protein structure analysis were done using Molecular Operating Environment (MOE) and Pymol. The structures were energy minimized prior to ligand docking simulations. All amino acids in the $5 \AA$ radius of the catalytic residues were identified as potential targets for mutation. Mutations of specific amino acids and combinatorial mutations were constructed in silico and stored as separate structure files for further analysis, creating an in silico library. First, a docking simulation was carried out with its native substrate- 1,2-PD and critically analyzed in comparison to the known substrate bound complex (PDBid: 1UC5). This ensured that the docking simulation predicted similar ligand interactions as previously determined from crystal structures. Further docking simulations were then carried out using the native protein and the engineered proteins to study the interaction of 1,2,4-BTO with the active site residues. The orientations of substrates were analyzed, their interactions with critical active site residues were studied and distances were calculated. 


\section{Results}

\subsection{Native dehydratase possesses nominal activity toward 1,2,4-butanetriol}

Diol dehydratases are commercially important enzymes which have been successfully utilized for the production of high value commodities like 1,3-propanediol from glycerol (Raynaud et al., 2003), 1-propanol from 1,2-PD (Jain and Yan, 2011), 2-butanone from meso-2,3-butanediol (Yoneda et al., 2014). In our previous work, we improved the biocatalytic efficiency of $K$. oxytoca diol dehydratase toward its native substrate (1,2-PD) by linking its 3 subunits to create a fusion protein (encoded by $p p d A-C-B$ ) (Jain et al., 2014). Here, we selected this fusion dehydratase for rational engineering approaches to dehydrate a non-native $\mathrm{C} 4$ triol, as it would be congenial to purification and characterization studies. We performed in vitro enzyme assays by coupling the dehydration activity of this native fusion dehydratase with the subsequent reduction activity of alcohol dehydrogenase (encoded by $y q h D$ ).

As seen from Table 2, native fusion diol dehydratase's catalytic activity was determined to be 32 fold lower toward 1,2,4-BTO $\left(k_{\text {cat }}=17.08 \mathrm{~min}^{-1}\right)$ as compared to its catalytic activity toward 1,2PD $\left(k_{c a t}=549.56 \mathrm{~min}^{-1}\right)$. In order to then evaluate its catalytic efficiency for in vivo production of 1,4-BDO we performed whole cell bioconversion studies. As seen from Fig. 2A, no detectable production of 4-hydroxybutrylaldehyde or 1,4-BDO was observed from cultures fed with 1,2,4BTO, even though activity toward 1,2,4-BTO was detected in vitro. The native dehydratase completely converted 1,2-PD in whole cell conversion studies, as indicated by 1-propanol production (Jain et al., 2014), validating our in vitro studies (Fig. 2B). Hence, to promote 1,4- 
BDO biosynthesis we pursued the enhancement in dehydratase's activity toward 1,2,4-BTO via rational engineering approaches encouraged by its inherent nominal activity.

\subsection{Improving resistance to substrate inhibition alone does not facilitate bio-catalysis}

The activity of $K$. oxytoca diol dehydratase is known to be influenced by the number of hydroxyl groups present in the substrate. High catalytic efficiency is exhibited toward 1,2-PD (a C3 diol), while limited catalytic efficiency is observed toward glycerol (a C3 triol) due to substrate inhibition (Yamanishi et al., 2012). An approach to address this inhibition has been developed from studying the protein-substrate complex, where it was inferred that the interaction of the C3 hydroxyl group of glycerol with S301 leads to inhibition of catalysis (Doitomi et al., 2012). Recently, it has been shown that the mutation of S301 to A301 prevents this interaction with glycerol, thereby making it more resistant to substrate inhibition (Yamanishi et al., 2012). Based on these findings we speculated that a similar substrate inhibition by a $\mathrm{C} 4$ triol $(1,2,4-\mathrm{BTO})$ was plausible. Hence, we hypothesized that by improving substrate inhibition resistance toward 1,2,4-BTO, desired bio-catalysis would be promoted. We then tested this hypothesis via whole cell bio-catalysis experiments.

The biocatalytic efficiency of S301A mutant toward 1,2,4-BTO, glycerol and 1,2-PD were tested. Contrary to our expectation, no detectable quantities of products were observed from cultures fed with 1,2,4-BTO (Fig. 2A). Furthermore, it was seen that the S301A mutant dehydratase showed a noticeable increase in resistance toward substrate inhibition by glycerol, consistent with previous studies (Yamanishi et al., 2012); leading to 1,3-propanediol production at $0.13 \mathrm{~g} / \mathrm{L}$ as compared 
to $0.04 \mathrm{~g} / \mathrm{L}$ from the native dehydratase (Fig. 2C). As seen from Fig. 2B, the native dehydratase completely converted all of the 1,2-PD, while the S301A mutant dehydratase showed a minor decrease in conversion efficiency toward 1,2-PD leading to 1-propanol production at $0.70 \mathrm{~g} / \mathrm{L}$ as compared to $0.81 \mathrm{~g} / \mathrm{L}$ from native dehydratase. We then speculated that although improvement in resistance toward a C3 triol substrate inhibition was achieved, it may be difficult to accommodate a C4 triol in the catalytic site of diol dehydratase, since 1,2,4-BTO is a larger substrate.

\subsection{Promoting substrate accommodation enhances bio-catalysis toward 1,2,4-butanetriol}

We then calculated the space in the catalytic pocket by measuring the distance between Q336 and Q296. We chose Q336 as a candidate for mutation to a smaller residue. Alanine was chosen as the substitute over glycine, as alanine's side chain is comparatively less reactive (Betts and Russel, 2003; Robinson and Sauer, 1998). By mutating Q336 to A336 we noticed an increase in this distance from $7.93 \AA$ to $8.47 \AA$ via in silico analysis (Fig. 3). We speculated that this would allow more space for the larger substrate to be accommodated. Such an increase in the space of the catalytic pocket due to mutation of Q336 to A336 has also been observed in previous studies (Yamanishi et al., 2012). Hence, we hypothesized that mutating Q336 to A336 would result in the desired dehydration activity due to accommodation of 1,2,4-BTO in the active site. In order to test this hypothesis, we created Q336A mutant dehydratase and performed whole cell biocatalysis experiments. As seen from Fig. 2A, the mutation of Q336 to A336 resulted in achieving detectable bio-catalysis toward 1,2,4-BTO, producing 1,4-BDO at $56 \mathrm{mg} / \mathrm{L}$. However, majority of the 1,2,4-BTO remained unconverted. Since no 4-hydroxybutrylaldehyde was detected, this 
result indicated that the native alcohol dehydrogenase activity of $E$. coli was sufficient to reduce 4-hydroxybutrylaldehyde to 1,4-BDO. Hence, we concluded that overexpression of a dehydrogenase was not necessary for the reduction of 4-hydroxybutrylaldehyde to 1,4-BDO. Interestingly, E. coli cultures expressing the Q336A mutant dehydratase exhibited a further reduction in conversion efficiency toward the native substrate 1,2-PD, indicated by a decrease in 1-propanol production to $0.29 \mathrm{~g} / \mathrm{L}$ (Fig. 2B). We also studied the activity of Q336A mutant dehydratase toward glycerol, and found a marginal increase in 1,3-propanediol production to $0.06 \mathrm{~g} / \mathrm{L}$ as compared to the native dehydratase $(0.04 \mathrm{~g} / \mathrm{L})$, while lower than that of S301A mutant (0.13 g/L) (Fig. 2C).

Next, we performed docking simulations to further analyze the interaction of 1,2,4-BTO with the Q336A mutant dehydratase. Docking simulations with Q336A mutant revealed that an interaction between S301 and 1,2,4-BTO was highly likely. We then hypothesized that by creating a S301AQ336A mutant, the catalytic efficiency toward 1,2,4-BTO may be further increased due to: (a) prevention of interaction between the $\mathrm{C} 4$ hydroxyl group of 1,2,4-BTO with S301 and, (b) promotion of substrate accommodation. In order to test this hypothesis, we constructed S301A Q336A mutant diol dehydratase and performed whole cell bio-catalysis experiments. As seen from Fig. 2A, biocatalytic efficiency toward 1,2,4-BTO was further improved as indicated by increase in 1,4-BDO production to about $145 \mathrm{mg} / \mathrm{L}$. Furthermore, we observed an even greater decrease in conversion efficiency toward 1,2-PD, indicated by a decrease in 1-propanol production to $0.13 \mathrm{~g} / \mathrm{L}$. While, improvement in resistance to substrate inhibition toward the $\mathrm{C} 3$ triol (glycerol) was found to be similar ( $0.05 \mathrm{~g} / \mathrm{L}$ 1,3-propanediol) to the 
native dehydratase (0.04 g/L 1,3-propanediol) (Fig. 2C). We then proceeded to characterize the in vitro catalytic efficiency of S301AQ336A mutant dehydratase via coupled enzyme assays using the purified protein. As seen from Table 2, activity toward 1,2,4-BTO was increased due to S301AQ336A mutations $\left(K_{\text {cat }}=76.21 \mathrm{~min}^{-1}\right)$, as compared to the native dehydratase $\left(K_{\text {cat }}=\right.$ 17.08 $\left.\mathrm{min}^{-1}\right)$. Consistent with our observations from whole cell bio-catalysis experiments, activity $\left(K_{\text {cat }}\right)$ toward native substrate 1,2-PD was decreased from $549.56 \mathrm{~min}^{-1}$ to $39.49 \mathrm{~min}^{-1}$. Overall, predominant rational design strategies (substrate accommodation and preventing undesired interaction) improved catalysis toward 1,2,4-butanetriol, however at low biocatalytic efficiency.

\subsection{Promoting the maintenance of O1-M ion distance improves activity toward 1,2,4-butanetriol}

In order to further improve catalytic activity toward 1,2,4-BTO, we used the knowledge of its reaction mechanism as a guiding principle to enhance non-native dehydratase based catalysis. The complex catalytic mechanism of diol dehydratase with 1,2-PD has been studied, where it was found that the binding of the substrate in coordination with the metal ion initiates catalysis via the homolytic cleavage of Co-C bond (of adenosylcobalamin) (Shibata et al., 2002). The resultant adenosyl radical abstracts a hydrogen atom from the $\mathrm{C} 1$ position of the substrate, thus creating a 1,2 diol radical. Next, the $\mathrm{OH}$ group from $\mathrm{C} 2$ of the substrate migrates to the $\mathrm{C} 1$ position, creating a 1,1 diol radical. With this, the 1,1 diol radical back abstracts the hydrogen atom from 5'-deoxyadenosine, producing a 1,1 diol product and regenerating the adenosyl radical. With this, the loss of a water molecule leads to the product aldehyde (Shibata et al., 2002). 
The metal ion plays an essential role in catalysis by (a) holding the substrate in the favorable orientation leading to initiation of catalysis, (b) allowing hydrogen abstraction from the substrate and $\mathrm{OH}$ group migration (Kamachi et al., 2011). It was determined that the distance between the metal ion and $\mathrm{O} 1$ of 1,2-PD was $2.5 \AA$, representative of an optimal distance for catalysis to proceed (Kamachi et al., 2011). From these studies we inferred that a distance close to $2.5 \AA$ between the metal ion in the active site and $\mathrm{O} 1$ of the substrate is critical to catalysis, as an increased distance may not be thermodynamically favorable to catalysis (hydrogen abstraction, hydrogen back-abstraction or $\mathrm{OH}$ group migration) leading to possible substrate inhibition. Hence, we hypothesized that rational engineering approaches may help promote a substrate orientation and position which is thermodynamically more favorable to catalysis ( $\mathrm{O} 1$ of substrate being closer to $2.5 \AA$ from metal ion).

It is commonly known that a substrate's orientation in the catalytic pocket can be influenced by residues in close proximity; we hypothesized that these interactions can be availed to persuade desired catalysis (by altering the distance between $\mathrm{O} 1$ of substrate and metal ion). We developed an in silico screening approach to (a) identify prospective targets within the $5 \AA$ radius that would influence substrate orientation, (b) serve as a screening platform to study the influence of substitutions on substrate $(1,2,4-\mathrm{BTO})$ orientation and distance from metal ion. Residues directly involved in catalysis were first eliminated as candidates (Q141, E170, E221, H143, Q296, D335 and S362) (Kamachi et al., 2011). T222, V300 and F374, were identified as potential targets initially, as they are within the $5 \AA$ radius, and not involved in catalysis directly. By performing 
docking studies, we evaluated the effect of substituting the three target residues to other amino acids and determined that the mutation of V300 led to a more pronounced effect on substrate position. This observation is also supported by previous studies speculating that V300 may play a role in distinguishing substrate conformations due to steric repulsion (Doitomi et al., 2014).

Next, we performed a systematic study in silico, by mutating V300 to different amino acids with hydrophobic side chains based upon the S301AQ336A mutant dehydratase. This was done in order to (a) maintain a relatively similar hydrophobic environment in the catalytic pocket, (b) promote the effects of steric hindrance on substrate position. Docking simulations were carried out for each case, and the distance between the metal ion and O1 of 1,2,4-BTO were calculated for enzyme substrate complexes with the lowest energy; serving as a screening criteria for identification of candidate mutants (Table 3).

This study indicated a strong correlation between the choice of amino acid substitution and the distance between $\mathrm{O} 1$ of 1,2,4-BTO and metal ion of the lowest energy complexes. It was seen that substitution of V300 with A300, M300 or F300 greatly influenced this distance, possibly due to steric repulsion (Table 3). It was observed that the substitution of V300 with M300 resulted in maintaining a distance of $2.63 \AA$ between O1 of 1,2,4-BTO and the metal ion (Fig. 4), while substitution with A300 or F300 increased this distance to $7.61 \AA$ or $8.32 \AA$ respectively (Table 3). We speculate that substitution with methionine maybe more likely to favor catalysis as (a) it's side chain is relatively more flexible than phenylalanine's, (b) methionine possesses a relatively less reactive side chain like alanine, (c) may promote a more pronounced steric hindrance effect 
toward 1,2,4-BTO, as it is bulkier than alanine (Betts and Russel, 2003). We also validated our docking studies by superimposing 1,2-PD obtained from crystal structure (PDBid:1UC5) with the docked 1,2,4-BTO (Fig. S1 in supplementary materials).

Next, we created three diol dehydratase variants (S301AQ336AV300A, S301AQ336AV300M and S301AQ336AV300F) and tested their activities toward 1,2,4-BTO via whole cell biocatalysis studies. As seen from Fig. 2A, the S301AQ336AV300M mutant further promoted catalysis as expected, and increased 1,4-BDO titer to nearly $220 \mathrm{mg} / \mathrm{L}$. No improvement in 1,4BDO production was observed from cultures expressing the S301AQ336AV300A or S301AQ336AF300 mutants. Interestingly, these mutants showed similar activity toward 1,2-PD and glycerol as the S301AQ336A mutant. Hence, the combinatorial mutation of Q336A, S301A and $\mathrm{V} 300 \mathrm{M}$ resulted in promoting catalysis toward the desired $\mathrm{C} 4$ triol.

We then characterized the in vitro catalytic efficiency of this S301AQ336AV300M mutant dehydratase via coupled enzyme assays. As seen from Table 2, activity of S301AQ336AV300M dehydratase toward 1,2,4-BTO $\left(K_{\text {cat }}=83.14 \mathrm{~min}^{-1}\right)$ was nearly 5 fold higher as compared to the native dehydratase $\left(K_{\text {cat }}=17.08 \mathrm{~min}^{-1}\right)$. Similar to the S301AQ336A mutant, the activity of S301AQ336AV300M mutant dehydratase toward 1,2-PD was decreased $\left(K_{\text {cat }}=38.11 \mathrm{~min}^{-1}\right)$. Additionally, we tested T222 or F374 mutations as control for 1,4-BDO production, via whole cell bio-catalysis studies. As expected, we were not able to identify any additional mutants leading to improved production of 1,4-BDO as compared to S301AQ336A mutant dehydratase (Fig. S2 in supplementary materials). Furthermore, we evaluated if the co-expression of 
reactivation factors $(d d r A B)$ would improve 1,4-BDO production, since its co-expression has been shown to improve dehydratase's resistance toward substrate inhibition by glycerol (Shibata et al., 2005). We performed whole cell conversion studies by feeding 1,2,4-BTO or glycerol as a control. It was observed that dehydratase's resistance toward substrate inhibition by glycerol was improved (indicated by improvement in 1,3-propanediol production), consistent with previous studies (Shibata et al., 2005). However, no noticeable improvement in 1,4-BDO production was observed from native or mutant dehydratases when co-expressed with reactivation factors.

\subsection{De novo biosynthesis of 1,4-butanediol from xylose}

After in vitro characterization of dehydratase and its mutants, we pursued the de novo biosynthesis of 1,4-BDO from xylose. To achieve this, we first adapted and engineered the 1,2,4BTO biosynthesis pathway from xylose (Abdel-Ghany et al., 2013; Niu et al., 2003; Valdehuesaa et al., 2014). It has been previously shown that the introduction of Caulobacter crescentus xylose dehydrogenase (encoded by $x y l B C$ ) and xylonic acid dehydratase (encoded by $x y l D$ ) as well as Lactococcus lactis keto acid decarboxylase (encoded by kivD) in E. coli enables the production of 1,2,4-BTO from xylose (Niu et al., 2003; Tai et al., 2016). In this pathway, xylose is catalyzed to xylonic acid by the action of $C$. crescentus xylose dehydrogenase, following which the activity of $C$. crescent xylonic acid dehydratase results in the formation of 3-deoxy-glycero-pentulosonic acid. Next, 3-deoxy-glycero-pentulosonic acid is decarboxylated to 3,4-dihydroxy-butanal by the action of $L$. lactis keto acid decarboxylase. 1,2,4-BTO is then produced by the action of native alcohol dehydrogenase $(y q h D)$ on 3,4-dihydroxy-butanal (Niu et al., 2003; Valdehuesaa et al., 2014) (Fig. 1). 
We initially performed a systematic approach to improve the production of 1,2,4-butanetriol. The complete 1,2,4-BTO pathway $(x y l B C, x y l D, k i v D, y q h D)$ was first introduced into $E$. coli strain BW25113. As seen from Fig. 5, 1,2,4-BTO production was achieved at $224 \mathrm{mg} / \mathrm{L}$. Next, JW171 was constructed to disrupt major carbon competing pathway for xylose utilization (pentose phosphate pathway via the action of xylose isomerase, encoded by $x y l A$ ), the titer of 1,2,4-BTO was increased to $437 \mathrm{mg} / \mathrm{L}$. In order to further prevent the loss of carbon to major competing pathways, we constructed E. coli strain JW173 with $x y l A$ and $y j h H$ gene disruptions and E. coli strain JW175 with $x y l A$ and $y a g E$ gene disruptions (Fig. 1). As seen from Fig. 5, 1,2,4-BTO production was improved to $730 \mathrm{mg} / \mathrm{L}$ or $543 \mathrm{mg} / \mathrm{L}$ from strains JW173 and JW175 expressing the complete 1,2,4-BTO pathway respectively. Finally, to maximize the utilization of carbon toward 1,2,4-BTO production, we constructed an E. coli mutant strain with $x y l A, y j h H$ and $y a g E$ gene disruptions (JW177). We hypothesized that in doing so, nearly all of the xylose would be utilized for 1,2,4-butanetriol production. We then performed shake flask experiments using strain JW177 transformed with plasmids expressing the complete 1,2,4-BTO pathway. It was seen that strain JW177 expressing the complete 1,2,4-BTO pathway further improved 1,2,4-BTO production to $1506 \mathrm{mg} / \mathrm{L}$ (Fig. 5). Hence, by reducing loss of carbon to native metabolism the production of 1,2,4-BTO was enhanced.

After achieving 1,2,4-BTO biosynthesis at $1506 \mathrm{mg} / \mathrm{L}$, we proceeded to over-express diol dehydratase in addition to $1,2,4-\mathrm{BTO}$ pathway for 1,4-BDO production. To do this, we used the best performed strain JW177 and transformed it with pJW60 and pJW84 or pJW85 or pJW86 
(plasmids carrying $x y l D$, kivD along with the native or S301AQ336A mutant or S301AQ336AV300M mutant diol dehydratase respectively). After 48 hours of shake flask studies it was seen that the overexpression of native dehydratase along with the 1,2,4-BTO pathway did not produce any detectable quantities of 1,4-BDO (Fig. 6). However, the overexpression of S301AQ336A mutant dehydratase along with the 1,2,4-BTO pathway resulted in the de novo biosynthesis of 1,4-BDO at $151 \mathrm{mg} / \mathrm{L}$. About $1261 \mathrm{mg} / \mathrm{L}$ of 1,2,4-BTO was left unconverted from this strain. We then tested the effect of overexpressing the S301AQ336AV300M mutant diol dehydratase along with the 1,2,4-BTO pathway on 1,4-BDO biosynthesis. As expected, 1,4-BDO production was further improved, achieving $209 \mathrm{mg} / \mathrm{L}$ from strain RJ177. About $1209 \mathrm{mg} / \mathrm{L}$ of 1,2,4-BTO was left unconverted at the end of the study from this strain (Fig. 6). These results supported the observed activity of diol dehydratase and its mutants as inferred from whole cell bio-catalysis and in vitro assay experiments. Hence, de novo biosynthesis of 1,4-BDO was achieved directly from xylose at $209 \mathrm{mg} / \mathrm{L}$. 


\section{Discussion}

In this study, we demonstrated the prudent utilization of rational engineering approaches to engineer a diol dehydratase and achieve non-native catalysis. The use of in silico screening approaches via docking simulations led to the identification of critical amino acid residues, and also the influence of the substitutions without requiring a high-throughput screening platform. We demonstrated that pursuing substrate accommodation led to enabling catalytic ability toward the non-native substrate, while, further improvement in desired catalysis is accomplished via conception of rational design approaches reliant on the reaction mechanism. This two-fold approach reinforced the utilization of rationally conceived hypotheses to enhance non-native catalysis.

These efforts enabled the expansion of the 1,2,4-BTO biosynthesis pathway to 1,4-BDO. First, we enhanced 1,2,4-BTO production from $224 \mathrm{mg} / \mathrm{L}$ to $1506 \mathrm{mg} / \mathrm{L}$. With this, the expression of the complete pathway enabled 1,4-BDO production at $209 \mathrm{mg} / \mathrm{L}$. It was observed that although 1,2,4-BTO production was enhanced by strain engineering, its conversion to 1,4-BDO was not improved conjointly. Hence, before proceeding to scale-up studies, further efforts to improve 1,4-BDO production may include enhancing dehydratase activity toward 1,2,4-BTO via directed evolution strategies and metabolic engineering to increase xylose uptake. Overall, we report that by rationally redesigning diol dehydratase biological production of 1,4-BDO was enabled through a novel metabolic route from xylose. 


\section{Acknowledgements}

This work was supported by National Science Foundation (Grant Number 1349499). We would also like to thank the National Natural Science Foundation of China $(21376017,21406010$, 21636001), the Programme of Introducing Talents of Discipline to Universities (“111” project, B13005), the Program for Changjiang Scholars and Innovative Research Team in Universities in China (No. IRT13045). 
Table 1. List of strains and plasmids used in this study.

\begin{tabular}{|c|c|c|}
\hline Strain & Genotype & Reference \\
\hline E. coli BW25113 & $\begin{array}{l}F-, \Delta(\operatorname{araD}-a r a B) 567, \Delta l a c Z 4787(: \because r r n B-3), \\
\lambda-, r p h-1, \Delta(r h a D-r h a B) 568, h s d R 514\end{array}$ & Yale CGSC \\
\hline E. coli XL1-Blue & $\begin{array}{l}\text { recA1 } \\
\text { endA1gyrA96thi-1hsdR17supE44relA1lac } \\
\text { [F'proAB lacIqZDM15Tn10 (TetR)] }\end{array}$ & Stratagene \\
\hline $\begin{array}{l}\text { E. coli BL21 Star } \\
\text { (DE3) }\end{array}$ & $F^{-}$ompT hsd $S_{B}\left(r_{B^{-}} m_{B}^{-}\right)$gal dcm (DE3) & Invitrogen \\
\hline JW3537-1 & BW25113 $\Delta x y l A:: k a n$ & Yale CGSC \\
\hline JW5775-2 & BW25113 $\Delta y j h H:: k a n$ & Yale CGSC \\
\hline JW0261-1 & BW25113 syagE::kan & Yale CGSC \\
\hline JW171 & BW25113 $\Delta x y l A$ & Unpublished \\
\hline JW173 & BW25113 $\Delta x y l A \Delta y j h H$ & Unpublished \\
\hline JW175 & BW25113 $\Delta x y l A \Delta y a g E$ & Unpublished \\
\hline JW177 & BW25113 $\Delta x y l A \Delta y a g E \Delta y j h H$ & Unpublished \\
\hline Plasmid & Description & Reference \\
\hline pZE12- luc & pLlacO-1; luc; ColE1 ori; $A m p^{R}$ & $\begin{array}{l}\text { (Lutz and Bujard, } \\
\text { 1997) }\end{array}$ \\
\hline pCS27 & pLlacO-1; p15A ori; Kan $^{R}$ & (Shen and Liao, 2008) \\
\hline pETDuet-1 & $\begin{array}{l}\text { two T7 promoters; two MCS; } p B R 322 \text { ori; } \\
A m p^{R}\end{array}$ & Novagen \\
\hline pJW79 & Fusion $p p d A-C-B$ cloned into pZE12-luc & This study \\
\hline pRJ75 & $d d r A B$ from $K$. oxytoca cloned into pCS 27 & This study \\
\hline
\end{tabular}




\begin{tabular}{|c|c|c|}
\hline pJW80-83, & Fusion $p p d A-C-B$ mutants cloned into & This study \\
\hline pRJ111-116, & pZE12-luc & \\
\hline \multicolumn{3}{|l|}{ pRJ161-167 } \\
\hline pRJ84 & Fusion $p p d A-C-B$ cloned into pETDuet-1 & This study \\
\hline pRJ91 & $\begin{array}{l}\text { Fusion } p p d A-C-B \text { S301AQ336A cloned into } \\
\text { pETDuet-1 }\end{array}$ & This study \\
\hline pRJ170 & $\begin{array}{l}\text { Fusion } p p d A-C-B \text { S301AQ336AV300M } \\
\text { cloned into pETDuet-1 }\end{array}$ & This study \\
\hline pRJ122 & $y q h D$ from $E$. coli cloned into pETDuet-1 & This study \\
\hline pJW60 & $\begin{array}{l}x y l B, x y l C \text { from } C \text {. crescentus, } y q h D \text { from } E \text {. } \\
\text { coli cloned into pCS } 27\end{array}$ & This study \\
\hline pJW76 & $\begin{array}{l}x y l D \text { from } C \text {. crescentus and kivD from } L . \\
\text { lactis cloned into pZE12-luc }\end{array}$ & Unpublished \\
\hline pJW84 & $\begin{array}{l}x y l D \text { from } C \text {. crescentus, kivD from } L \text {. lactis } \\
\text { and } p p d A-C-B \text { from } K \text {. oxytoca cloned into } \\
\text { pZE12-luc }\end{array}$ & This study \\
\hline pJW85 & $\begin{array}{l}x y l D \text { from } C \text {. crescentus, } k i v D \text { from } L \text {. lactis } \\
\text { and } p p d A-C-B \text { S301AQ336A from } K \text {. } \\
\text { oxytoca cloned into pZE12-luc }\end{array}$ & This study \\
\hline pJW86 & $\begin{array}{l}x y l D \text { from } C \text {. crescentus, } k i v D \text { from L. lactis } \\
\text { and } p p d A-C-B \text { S301AQ336AV300M from } K \text {. }\end{array}$ & This study \\
\hline & oxytoca cloned into pZE12-luc & \\
\hline
\end{tabular}


Table 2. Results of in vitro characterization of native and mutant diol dehydratases via coupled enzyme assay.

\begin{tabular}{cccc}
\hline Dehydratase & Substrate & $\boldsymbol{K}_{\text {cat }}\left(\mathbf{m i n}^{-1}\right)$ & $\boldsymbol{K}_{\boldsymbol{m}}(\mathbf{m M})$ \\
\hline Native & $1,2,4-\mathrm{BTO}$ & $17.08 \pm 0.09$ & $2.61 \pm 0.05$ \\
S301AQ336A & $1,2,4-\mathrm{BTO}$ & $76.21 \pm 7.62$ & $7.42 \pm 1.77$ \\
S301AQ336AV300M & $1,2,4-\mathrm{BTO}$ & $83.14 \pm 3.46$ & $7.34 \pm 0.80$ \\
Native & $1,2-\mathrm{PD}$ & $549.56 \pm 81.69$ & $7.90 \pm 2.74$ \\
S301AQ336A & $1,2-\mathrm{PD}$ & $39.49 \pm 0.69$ & $3.16 \pm 0.32$ \\
S301AQ336AV300M & $1,2-\mathrm{PD}$ & $38.11 \pm 1.39$ & $3.50 \pm 0.40$ \\
\hline
\end{tabular}


Table 3. Results of docking simulations with ( $\mathrm{O} 1$ of 1,2,4-BTO and metal ion) distance calculated as a result of influence on substrate position based upon amino acid substitutions.

\begin{tabular}{cccc}
\hline Dehydratase & Substrate & O1-M & Reference \\
& & Distance $(\AA)$ & \\
\hline S301AQ336A & $1,2,4-\mathrm{BTO}$ & 7.19 & Docking/ This study \\
S301AQ336AV300A & $1,2,4-\mathrm{BTO}$ & 7.61 & Docking/ This study \\
S301AQ336AV300M & $1,2,4-\mathrm{BTO}$ & 2.63 & Docking/ This study \\
S301AQ336AV300F & $1,2,4-\mathrm{BTO}$ & 8.32 & Docking/ This study \\
\hline
\end{tabular}




\section{Figure Legends}

Fig. 1. Schematic representation of a novel 1,4-butanediol pathway from xylose and native competing pathways in E. coli. Genes: $x y l A$ : encoding xylose isomerase; $x y l B C$ : encoding xylose dehydrogenase; $x y l D$ : encoding xylonate dehydratase; kivD: encoding alpha-ketoisovalerate decarboxylase; $y q h D$ : encoding alcohol dehydrogenase; $p p d A-C-B$ : encoding diol dehydratase; yagE or $y j h H$ : encoding aldolase.

Fig. 2. Results of whole cell bio-catalysis experiments using native and mutant dehydratases. (A) 1,4-Butanediol produced from whole cell bio-catalysis experiments by feeding 1,2,4-butanetriol; (B) 1-Propanol produced from whole cell bio-catalysis experiments by feeding 1,2-propanediol. The data were generated from three independent experiments. (C) 1,3-Propanediol produced from whole cell bio-catalysis experiments by feeding glycerol. The data were generated from three independent experiments, Error bars represent s.d. values.

Fig. 3. Representation of the catalytic pocket of native and Q336A mutant dehydratase. Distances were calculated in $\AA$ between Q296 and Q336/A336 using existing diol dehydratase crystal structure (PDBid: 1UC5).

Fig. 4. Representation of 1,2,4-butanetriol's interaction in the catalytic pocket of Q336AS301A V300M mutant diol dehydratase. Distance was calculated in $\AA$ between the metal ion and $\mathrm{C} 1 \mathrm{OH}$ of 1,2,4-butanetriol as obtained from docking studies. 
Fig. 5. Results of shake flask studies for de novo production of 1,2,4-butanetriol from xylose using engineered E. coli strains. The plasmids JW60 and JW76 were co-transformed into those strains. The data were generated from three independent experiments. Error bars represent s.d. values.

Fig. 6. Results of shake flask studies for de novo production of 1,4-butanediol from xylose using engineered E. coli strains. The data were generated from three independent experiments. Error bars represent s.d. values. 


\section{References}

Abdel-Ghany, S.E., Day, I., Heuberger, A.L., Broeckling, C.D., Reddy, A.S., 2013. Metabolic engineering of Arabidopsis for butanetriol production using bacterial genes. Metab. Eng. 20, 109-20.

Ajikumar, P.K., Xiao, W.H., Tyo, K.E., Wang, Y., Simeon, F., Leonard, E., Mucha, O., Phon, T.H., Pfeifer, B., Stephanopoulos, G., 2010. Isoprenoid pathway optimization for taxol precursor overproduction in Escherichia coli. Science. 330, 70-4.

Atsumi, S., Hanai, T., Liao, J.C., 2008. Non-fermentative pathways for synthesis of branchedchain higher alcohols as biofuels. Nature. 451, 86-89.

Avalos, J.L., Fink, G.R., Stephanopoulos, G., 2013. Compartmentalization of metabolic pathways in yeast mitochondria improves the production of branched-chain alcohols. Nat. Biotechnol. 31, 335-41.

Betts, M.J., Russel, R.B., 2003. Amino acid properties and consequences of substitutions. In: Barnes, M. R., Gray, I. C., Eds.), Bioinformatics for Geneticists. John Wiley \& Sons, Ltd., Chichester, U. K. , pp. 289-316.

Datsenko, K.A., Wanner, B.L., 2000. One-step inactivation of chromosomal genes in Escherichia coli K-12 using PCR products. Proc. Natl. Acad. Sci. U. S. A. 97, 6640-5.

Dietrich, J.A., McKee, A.E., Keasling, J.D., 2010. High-throughput metabolic engineering: advances in small-molecule screening and selection. Annu. Rev. Biochem. 79, 563-90.

Doitomi, K., Kamachi, T., Toraya, T., Yoshizawa, K., 2012. Inactivation mechanism of glycerol dehydration by diol dehydratase from combined quantum mechanical/molecular mechanical calculations. Biochemistry. 51, 9202-10. 
Doitomi, K., Tanaka, H., Kamachi, T., Toraya, T., Yoshizawa, K., 2014. Computational mutation design of diol dehydratase: catalytic ability toward glycerol beyond the wild-type enzyme. Bull. Chem. Soc. Jan. 87, 950-959.

Eiteman, M.A., Chastain, M.J., 1997. Optimization of the ion-exchange analysis of organic acids from fermentation. Anal. Chim. Acta. 338, 69-75.

Huo, Y.X., Cho, K.M., Rivera, J.G., Monte, E., Shen, C.R., Yan, Y., Liao, J.C., 2011. Conversion of proteins into biofuels by engineering nitrogen flux. Nat. Biotechnol. 29, 346-51.

Hwang, H.J., Park, J.H., Kim, J.H., Kong, M.K., Kim, J.W., Park, J.W., Cho, K.M., Lee, P.C., 2014. Engineering of a butyraldehyde dehydrogenase of Clostridium saccharoperbutylacetonicum to fit an engineered 1,4-butanediol pathway in Escherichia coli. Biotechnol. Bioeng. 111, 1374-1384.

Jain, R., Sun, X., Yuan, Q., Yan, Y., 2014. Systematically engineering Escherichia coli for enhanced production of 1,2-propanediol and 1-propanol. ACS Synth. Biol.

Jain, R., Yan, Y., 2011. Dehydratase mediated 1-propanol production in metabolically engineered Escherichia coli. Microb. Cell. Fact. 10.

Jang, Y.-S., Kim, B., Shin, J.H., Choi, Y.J., Choi, S., Song, C.W., Lee, J., Park, G.H., Lee, S.Y., 2012. Bio-based production of C2-C6 platform chemicals. Biotechnol. Bioeng. 109, 2437-2459.

Ji, X.-J., Huang, H., 2014. Bio-based butanediols production: the contributions of catalysis, metabolic engineering, and synthetic biology. In: Bisaria, V. S., Kondo, A., Eds.), Bioprocessing of Renewable Resources to Commodity Bioproducts. John Wiley \& Sons, Inc., Hoboken, NJ, USA, pp. 261-288. 
Jung, Y.K., Lee, S.Y., 2011. Efficient production of polylactic acid and its copolymers by metabolically engineered Escherichia coli. J. Biotechnol. 151, 94-101.

Kamachi, T., Doitomi, K., Takahata, M., Toraya, T., Yoshizawa, K., 2011. Catalytic roles of the metal ion in the substrate-binding site of coenzyme B12-dependent diol dehydratase. Inorg. Chem. 50, 2944-52.

Kan, S.J., Lewis, R.D., Chen, K., Arnold, F.H., 2016. Directed evolution of cytochrome c for carbon-silicon bond formation: Bringing silicon to life. Science. 354, 1048-1051.

Lan, E.I., Ro, S.Y., Liao, J.C., 2013. Oxygen-tolerant coenzyme A-acylating aldehyde dehydrogenase facilitates efficient photosynthetic n-butanol biosynthesis in cyanobacteria. Energy Environ. Sci. 6, 2672.

Lin, Y., Jain, R., Yan, Y., 2014. Microbial production of antioxidant food ingredients via metabolic engineering. Curr. Opin. Biotechnol 26, 71-78.

Liu, H., Lu, T., 2015. Autonomous production of 1,4-butanediol via a de novo biosynthesis pathway in engineered Escherichia coli. Metab. Eng. 29, 135-141.

Lutz, R., Bujard, H., 1997. Independent and tight regulation of transcriptional units in Escherichia coli via the LacR/O, the TetR/O and AraC/I1-I2 regulatory elements. Nucleic Acids Res. 25, 1203-1210.

Marcheschi, R.J., Gronenberg, L.S., Liao, J.C., 2013. Protein engineering for metabolic engineering: current and next-generation tools. Biotechnol J. 8, 545-55.

Nikel, P.I., Almeida, A.D., Giordano, A.M., Pettinari, M.J., 2010. Redox driven metabolic tuning: Carbon source and aeration affect synthesis of poly(3-hydroxybutyrate) in Escherichia coli. Bioeng. Bugs. 1, 291-295. 
Niu, W., Molefe, M.N., Frost, J.W., 2003. Microbial synthesis of the energetic material precursor 1,2,4-butanetriol. J. Am. Chem. Soc. 125, 12998-12999.

Ohta, K., Beall, D.S., Mejia, J.P., Shanmugam, K.T., Ingram, L.O., 1991. Genetic improvement of Escherichia coli for ethanol production: Chromosomal integration of Zymomonas mobilis genes encoding pyruvate decarboxylase and alcohol dehydrogenase II. Appl. Environ. Microbiol. 57, 893-900.

Raynaud, C., Sarcabal, P., Meynial-Salles, I., Croux, C., Soucaille, P., 2003. Molecular characterization of the 1,3-propanediol (1,3-PD) operon of Clostridium butyricum. Proc. Natl. Acad. Sci. U. S. A. 100, 5010-5.

Ro, D.K., Paradise, E.M., Ouellet, M., Fisher, K.J., Newman, K.L., Ndungu, J.M., Ho, K.A., Eachus, R.A., Ham, T.S., Kirby, J., Chang, M.C., Withers, S.T., Shiba, Y., Sarpong, R., Keasling, J.D., 2006. Production of the antimalarial drug precursor artemisinic acid in engineered yeast. Nature. 440, 940-3.

Robinson, C.R., Sauer, R.T., 1998. Optimizing the stability of single-chain proteins by linker length and composition mutagenesis. Proc. Natl. Acad. Sci. U. S. A. 95, 5929-34.

Savile, C.K., Janey, J.M., Mundorff, E.C., Moore, J.C., Tam, S., Jarvis, W.R., Colbeck, J.C., Krebber, A., Fleitz, F.J., Brands, J., Devine, P.N., Huisman, G.W., Hughes, G.J., 2010. Biocatalytic asymmetric synthesis of chiral amines from ketones applied to sitagliptin manufacture. Science. 329, 305-9.

Shen, C.R., Liao, J.C., 2008. Metabolic engineering of Escherichia coli for 1-butanol and 1propanol production via the keto-acid pathways. Metab. Eng. 10, 312-320. 
Shen, X., Lin, Y., Jain, R., Yuan, Q., Yan, Y., 2012. Inhibition of acetate accumulation leads to enhanced production of (R,R)-2,3-butanediol from glycerol in Escherichia coli. J. Ind. Microbiol. Biotechnol. 39, 1725-9.

Shibata, N., Masuda, J., Morimoto, Y., Yasuoka, N., Toraya, T., 2002. Substrate-induced conformational change of a coenzyme B12-dependent enzyme: crystal structure of the substrate-free form of diol dehydratase. Biochem. J. 41, 12607-17.

Shibata, N., Mori, K., Hieda, N., Higuchi, Y., Yamanishi, M., Toraya, T., 2005. Release of a damaged cofactor from a coenzyme B12-dependent enzyme: X-ray structures of diol dehydratase-reactivating factor. Structure. 13, 1745-54.

Shibata, N., Nakanishi, Y., Fukuoka, M., Yamanishi, M., Yasuoka, N., Toraya, T., 2003. Structural rationalization for the lack of stereospecificity in coenzyme B12-dependent diol dehydratase. J. Biol. Chem. 278, 22717-22725.

Sun, X., Shen, X., Jain, R., Lin, Y., Wang, J., Sun, J., Wang, J., Yan, Y., Yuan, Q., 2015. Synthesis of chemicals by metabolic engineering of microbes. Chem. Soc. Rev. 44, 3760-3785.

Tai, Y., Xiong, M., Jambunathan, P., Wang, J., Wang, J., Stapleton, C., Zhang, K., 2016. Engineering nonphosphorylative metabolism to generate lignocellulose-derived products. Nat. Chem. Biol. 12, 247-253.

Thomason, L.C., Costantino, N., Court, D.L., 2007. E. coli genome manipulation by P1 transduction. John Wiley \& Sons.

Valdehuesaa, K.N.G., Liua, H., Ramosa, K.R.M., Parka, S.J., Nisolaa, G.M., Leeb, W.-K., Chunga, W.-J., 2014. Direct bioconversion of d-xylose to 1,2,4-butanetriol in an engineered Escherichia coli. Process Biochem. 49, 25-32. 
Vemuri, G.N., Eiteman, M.A., Altman, E., 2002. Effects of growth mode and pyruvate carboxylase on succinic acid production by metabolically engineered strains of Escherichia coli. Appl. Environ. Microbiol. 68, 1715-1727.

Xiong, M., Schneiderman, D.K., Bates, F.S., Hillmyer, M.A., Zhang, K., 2014. Scalable production of mechanically tunable block polymers from sugar. Proc. Natl. Acad. Sci. U. S. A. $111,8357-62$.

Xu, P., Gu, Q., Wang, W., Wong, L., Bower, A.G., Collins, C.H., Koffas, M.A., 2013. Modular optimization of multi-gene pathways for fatty acids production in E. coli. Nat. Commun. 4, 1409 .

Yamanishi, M., Kinoshita, K., Fukuoka, M., Saito, T., Tanokuchi, A., Ikeda, Y., Obayashi, H., Mori, K., Shibata, N., Tobimatsu, T., Toraya, T., 2012. Redesign of coenzyme B(12) dependent diol dehydratase to be resistant to the mechanism-based inactivation by glycerol and act on longer chain 1,2-diols. FEBS J. 279, 793-804.

Yan, Y., Li, Z., Koffas, M.A., 2008. High-yield anthocyanin biosynthesis in engineered Escherichia coli. Biotechnol. Bioeng. 100, 126-40.

Yim, H., Haselbeck, R., Niu, W., Pujol-Baxley, C., Burgard, A., Boldt, J., Khandurina, J., Trawick, J.D., Osterhout, R.E., Stephen, R., Estadilla, J., Teisan, S., Schreyer, H.B., Andrae, S., Yang, T.H., Lee, S.Y., Burk, M.J., Van Dien, S., 2011. Metabolic engineering of Escherichia coli for direct production of 1,4-butanediol. Nat. Chem. Biol. 7, 445-52.

Yoneda, H., Tantillo, D.J., Atsumi, S., 2014. Biological production of 2-butanone in Escherichia coli. ChemSusChem. 7, 92-5. 